\title{
Comparison of the Effect of Vessel Size Imaging and Cerebral Blood Volume Derived from Perfusion MR Imaging on Glioma Grading
}

\author{
H.-Y. Kang, H.-L. Xiao, J.-H. Chen, Y. Tan, X. Chen, T. Xie, J.-Q. Fang, S. Wang, Y. Yang, and W.-G. Zhang
}

ON E

\begin{abstract}
BACKGROUND AND PURPOSE: Vascular proliferation is a major criterion for grading gliomas on the basis of histology. Relative cerebral blood volume can provide pathophysiologic information about glioma grading. Vessel size imaging, in some animals, can be used to estimate the microvascular caliber of a glioma, but its clinical use remains unclear. Herein, we aimed to compare the predictive power of relative cerebral blood volume and vessel size imaging in glioma grading, with grading based on histology.
\end{abstract}

MATERIALS AND METHODS: Seventy patients with glioma participated in the study; 30 patients underwent MR perfusion imaging with a spin-echo sequence and vessel size imaging with a gradient-echo and spin-echo sequence successively at 24-hour intervals before surgery. We analyzed the vessel size imaging values and relative cerebral blood volume of differently graded gliomas. The microvessel parameters were histologically evaluated and compared with those on MR imaging. The cutoff values of vessel size imaging and relative cerebral blood volume obtained from receiver operating characteristic curve analyses were used to predict glioma grading in another 40 patients.

RESULTS: Vessel size imaging values and relative cerebral blood volume were both increased in high-grade gliomas compared with low-grade gliomas $(P<.01)$. Moreover, vessel size imaging values had higher specificity and sensitivity in differentiating high-grade from low-grade gliomas compared with relative cerebral blood volume. In addition, a significant correlation was observed between vessel size imaging values and microvessel diameters $(r>0.8, P<.05)$ and between relative cerebral blood volume and microvessel area $(r=0.6579$, $P<$.05). Most important, the use of vessel size imaging cutoff values to predict glioma grading was more accurate (100\%) than use of relative cerebral blood volume (85\%) values.

CONCLUSIONS: Vessel size imaging can provide more accurate information on glioma grading and may serve as an effective biomarker for the prognosis of patients with gliomas.

ABBREVIATIONS: $\mathrm{HGG}=$ high-grade glioma; $\mathrm{LGG}=$ low-grade glioma; $\max =$ maximum; $\mathrm{MVA}=$ microvessel area; $\mathrm{MVD}=$ microvessel density; $\mathrm{rCBV}=$ relative cerebral blood volume; $\mathrm{SE}=$ spin-echo; $\mathrm{VSI}=$ vessel size imaging

G liomas with different grades have different clinical behaviors that determine treatment planning and patient prognosis in clinical practice. ${ }^{1,2}$ Biopsy or examination of tumor tissues from surgical resection specimens is the only method available to establish the final diagnosis of tumor grading in cases of malignancy; however, this procedure is invasive. ${ }^{3}$ With regard to

Received March 17, 2015; accepted after revision May 14.

From Departments of Radiology (H.-Y.K., J.-H.C., H.-L.X., Y.T., X.C., T.X., J.-q.F., W.-G.Z.) and Pathology (H.-L.X.), and State Key Laboratory of Trauma, Burns and Combined Injury (W.-G.Z.), Institute of Surgery Research, Daping Hospital, Third Military Medical University, Chongqing, China; and Departments of Radiology (S.W.) and Medicine (Y.Y.), Perelman School of Medicine, University of Pennsylvania, Philadelphia, Pennsylvania.

This study was supported by grants from Natural Science Foundation of China (No. 81271626), Natural Science Foundation Project of Chongqing (cstc2012jjB10028), and the Scientific Foundation of the Institute of Surgery Research, Daping Hospital, Third Military Medical University (No. 2014YLC03).

Please address correspondence to Wei-Guo Zhang, MD, Institute of Surgery Research, Daping Hospital, Third Military Medical University, Chongqing, 400042, China; e-mail: wgzhang01@163.com gliomas, tumor heterogeneity limits the ability of biopsies to accurately grade glioma tumors.

Functional MR imaging can provide quantitative or semiquantitative hemodynamic parameters, thereby allowing preoperative evaluation of the status of tumor angiogenesis, an important index in evaluating the grade of tumors and patient prognosis. ${ }^{4} \mathrm{CBV}$ maps, derived from MR imaging during the application of first-pass bolus-tracking analysis, provide complementary information in the diagnosis of tumor grade and the extent of malignancy. Several authors have found that maximal relative cerebral blood volume (rCBV) correlates with glioma grading. ${ }^{5-7}$ However, studies have shown that there were no significant differences in rCBV values between grade II and III glio-

\footnotetext{
- Indicates open access to non-subscribers at www.ajnr.org

Indicates article with supplemental on-line table and appendix.

Indicates article with supplemental on-line photo.

http://dx.doi.org/10.3174/ajnr.A4477
} 
mas ${ }^{8}$ or between grade III and IV gliomas. ${ }^{9}$ Moreover, a growing body of research has demonstrated the limited predictive power of rCBV to grade oligodendrogliomas, which tend to have high rCBVs regardless of glioma grade. Furthermore, oligodendrogliomas exhibit greater microvascular proliferation than astrocytomas $^{8,10-12}$; thus, rCBV may not be an ideal diagnostic tool for oligodendroglioma. ${ }^{8,13,14}$ Therefore, it is essential to explore an effective index for glioma grading that is independent of blood volume.

Vessel size imaging (VSI), which is obtained from the ratio of gradient-echo and spin-echo (SE) relaxation rate changes $\left(\triangle \mathrm{R} 2^{*}\right)$ $\triangle \mathrm{R} 2$ ) induced by intravascular superparamagnetic contrast agents, provides information on the average vessel size under certain conditions, such as the concentration of contrast agent, the main magnetic field, and TE. ${ }^{15}$ Recently, an increasing number of articles have reported that VSI can reflect the microvascular structures of tumors in animal models and strongly correlates with brain tumor grade. ${ }^{16,17}$ For example, Kiselev et al $^{18}$ reported that VSI increased the information value on brain histopathology obtained during dynamic perfusion measurements and that the mean vessel size correlated with the tumor type. Lemasson et al indicated that VSI in MR imaging estimates was closest to histology and provided complementary information on characterizing angiogenesis beyond fractioned blood volume in the rat brain tumor model. ${ }^{4}$ Although several studies have indicated that VSI had a high accuracy rate for the diagnosis of the degree of tumor malignancy in animal models, the predictive power of VSI in human gliomas remains largely unknown. ${ }^{16,18,19}$ To our knowledge, the comparison of VSI and $\mathrm{rCBV}$ in human glioma grading has not been addressed previously.

We designed this prospective study, in which the sensitivities and specificities of rCBV and VSI in distinguishing low-grade glioma (LGG) from high-grade glioma (HGG) were compared, and the final diagnosis of the grades of gliomas and the measurement of microvessel parameters were performed histologically on the basis of surgical resection specimens. The optimal cutoff values of rCBV and VSI were used to evaluate their diagnostic accuracy rates for predicting glioma grading.

\section{MATERIALS AND METHODS Patients and Samples}

We selected patients with suspected primary gliomas followed by undergoing prospective perfusion-weighted imaging between March 2013 and August 2014. Then, tumors were diagnosed and classified according to World Health Organization 2007 criteria as confirmed by surgery. Among the selected patients, at least 30 had undergone both SE-perfusion and VSI at 24- to 48-hour intervals; these patients were assigned to group 1, from which the optimal cutoff value of VSI and rCBV to distinguish HGG from LGG would be generated, respectively. Once the optimal cutoff values were obtained, we needed to evaluate their diagnostic accuracy rates in the grading of gliomas. Thus, we designed protocols for the enrollment of, at minimum, 40 patients with suspected primary gliomas, followed by those who underwent SE-perfusion (20 patients), assigned to group 2, or VSI (20 patients), assigned to group 3 (On-line Table 1). In our next step, we used the optimal cutoff values of rCBV and VSI to predict the glioma grading of patients in groups 2 and 3, prospectively. Finally, diagnostic accuracy was calculated by comparing calculated values with pathologic results.

The inclusion criteria were the following: 1) MR imaging examination before the histologic examination; 2) no therapy before the MR imaging examination; 3) gliomas confirmed histologically; and 4) patients in group 1, whose clinical symptoms occurred in lesser degrees, able to undergo both SE perfusion and VSI and agreeing to undergo perfusion twice. The exclusion criteria were the following: 1) patients older than 80 years of age; 2) gliomas with grade I and histologic results showing no gliomas or rare gliomas; 3 ) in group 1, patients not having enough time ( $>24$ hours) to have both SE perfusion and VSI before undergoing their operation; 4) contraindications to MR imaging; and 5) patients having false teeth, which would result in poor image quality. The study was approved by the local ethics committee at our institution, and all patients completed a written consent form.

\section{MR Imaging}

A 3T scanner (Magnetom Verio; Siemens, Erlangen, Germany) was used to acquire conventional MR imaging and DWI (details shown in the On-line Appendix) and spin-echo echo-planar perfusion scans. VSI was performed by using a 1.5T imager (Sigma HDx; GE Healthcare, Milwaukee, Wisconsin). The location line for all axial MR imaging was the central intercommissural (anterior/posterior commissure) line as previously described ${ }^{13,20}$ or parallel to this line.

Dynamic susceptibility contrast-enhanced perfusion MR imaging was performed by using the SE-EPI technique during administration of Gd-DTPA (Magnevist; Bayer HealthCare Pharmaceuticals, Wayne, New Jersey). The imaging parameters were as follows: TR/TE, $1500 / 30 \mathrm{~ms}$; flip angle, $90^{\circ}$; matrix size, $128 \times$ 128; NEX, 1.0; FOV, $23 \times 23 \mathrm{~cm}$. We selected 20 sections for perfusion imaging, in accordance with the T2-weighted imaging; at each section, 60 images were obtained. After 8 acquisitions, a bolus of gadobutrol ( $0.2 \mathrm{mmol}$ per kilogram of body weight) was injected at a rate of $3 \mathrm{~mL} / \mathrm{s}$, immediately followed by a $20-\mathrm{mL}$ bolus of saline at the same rate.

MR imaging data for VSI were acquired followed by intravenous injection of Gd-DTPA by using a gradient-echo and spinecho sequence, with the following acquisition parameters: TR, $1500 \mathrm{~ms}$; TE (gradient echo), $30 \mathrm{~ms}$; TE (SE), $100 \mathrm{~ms}$; flip angle, $90^{\circ}$; matrix size, $64 \times 64$; NEX, 1; FOV, $24 \times 24 \mathrm{~cm}$. Seven sections were selected across the tumor, with 120 images obtained at each section. In addition, VSI was obtained during injection of a bolus of $0.2 \mathrm{mmol} / \mathrm{kg}$ body weight of gadopentetate dimeglumine contrast agent at a rate of $3.5 \mathrm{~mL} / \mathrm{s}$ with a time delay of 18 seconds, followed by a $20-\mathrm{mL}$ bolus of saline.

\section{VSI Measurements}

The VSI images were analyzed by using an Advantage Workstation (Version 4.9; GE Healthcare) equipped with a dedicated software package (VSI; GE Healthcare). Using wide-bound or wholeobserved brain as the input function and the generation of contrast-enhancement time-signal intensity curves for the input function, we calculated $\triangle \mathrm{R} 2{ }^{\star} / \triangle \mathrm{R} 2$ according to the decay curve. 
On the basis of the known observation that $\triangle R 2^{\star} / \triangle R 2$ is a function of vessel size, ${ }^{21}$ VSI can be computed from this ratio according to the equation ${ }^{4,19}: \mathrm{VSI}=0.425(A D C / \gamma \triangle \times B 0)^{1 / 2} \times\left(\Delta \mathrm{R} 2^{*} /\right.$ $\Delta \mathrm{R} 2)^{3 / 2}$, where $A D C$ is the diffusion coefficient, by using 0.8 $\mu \mathrm{m}^{2} / \mathrm{ms}$ as analog data ${ }^{18} ; \gamma$ is the gyromagnetic ratio, the gyromagnetic ratio of hydrogen proton being $42.58 \mathrm{MHz} / \mathrm{T} ; \Delta \gamma$ is the change values in magnetization rate; and $B O$ is the stationary field, 1.5T. The software (VSI) generated color-coded VSI images, the threshold of which was adjusted to $0-80 \mu \mathrm{m}$ or $0-120$ $\mu \mathrm{m}$.

The preoperative and postoperative MR images were compared to outline the extent of surgery to increase the consistency between the regions of MR imaging and pathology measurements. A hand-drawn ROI of constant size $\left(56 \mathrm{~mm}^{2}\right)$ corresponding to the greatest visualized regions of perfusion was achieved by repeatedly moving the region in adjacent parts at least 5 times until the highest value was determined. We obtained approximately 10 values from at least 3 regions of the different crosssectional images, while avoiding the vessels and necrotic tissue, recording the mean and maximal $(\max )$ values of all data as the $\mathrm{VSI}_{\text {mean }}$ and VSI $\mathrm{max}_{\text {max }}$ values for each patient. Measurements were performed by 2 neuroradiologists (H.-Y.K. and J.-Q.F, with 7 and 6 years of experience, respectively). Each observer, who was blinded to the histopathologic diagnosis, conducted each measurement twice and independently.

\section{rCBV Measurements}

The original SE images were processed with a commercial software package (SyngoMMWP VE36A; Siemens) and were used to generate color-coded CBV maps. ${ }^{3,22,23}$ The enhanced T1weighted images were used as a guide for the location of the tumors to avoid the large vessels and necrotic tissue. The highest $\mathrm{CBV}$ values were obtained, and the $\mathrm{rCBV}_{\max }$ normalization to the contralateral unaffected white matter $\mathrm{CBV}$ value was based on previously published methods. ${ }^{1,23,24}$ Measurements were performed by 2 neuroradiologists (H.-Y.K. and J.-Q.F). Each ROI was independently checked for accuracy by another observer, and any changes were made by joint agreement. The size of the ROIs was kept constant (radius $=2.6 \mathrm{~mm}$ ). All analyses were performed without knowledge of tissue analyses.

\section{Immunohistology Analysis}

Each paraffin block from the 30 patients of group 1 was processed into a $4-\mu \mathrm{m}$-thick section, with 2-6 paraffin sections produced per patient. To measure the diameter of microvessels, microvessel area (MVA), and microvessel density (MVD) for grades II, III, or IV gliomas, we performed a histologic evaluation by immunohistochemistry for the CD34 antigen, which identified vascular endothelial cells as previously described. ${ }^{3,25}$ The reagent PV-6000-G Polymer Detection System for Immuno-Histologic Staining was bought from Beijing Zhong Shan-Golden Bridge Biologic Technology Company (Beijing, China). All tissue sections were digitized by using a fluorescence microscope (BX41; Olympus, Rungis, France) and the CellSens Standard software (http://www. scientific-computing.com/press-releases/product_details.php? product_id=809).

We examined entire sections at $\times 40$ magnification, covering an area of $1.308 \times 1.757 \mathrm{~mm} /$ field, to identify "hot spot" regions of microvascular diameter, MVA, and MVD, from which 5-10 hot spots were selected for imaging. ${ }^{26}$ By definition, the smallest countable blood vessels were used to estimate microvessel parameters. ${ }^{27}$ Microvascular diameter and MVA were measured at $\times 200$ magnification, and MVD, at $\times 100$ magnification. ${ }^{28}$ Quantitative microvascular analysis was performed by using Image-Pro Plus 5.0 software (http://www.mediacy.com/index.aspx?page= IPP), the microvessel diameter and MVA were determined by using the feret ( $\mathrm{min}$ ) and area (polygon) function, ${ }^{3}$ but the vessels with thick walls and lumens $>72 \mu \mathrm{m}$ were excluded. ${ }^{27}$ To minimize bias in the correlation between MR imaging and the histologic estimates, we analyzed the average diameter of all microvessels, MVD, and total area of all hot spot regions of each tissue section, and we compared sections from each patient to identify the highest values. Two experienced neuropathologists (H.-L.X and S.-G.F., with $>10$ years of experience) were blinded to the MR imaging results and completed the histologic measurements.

\section{Statistical Analysis}

Statistical analyses were performed by using commercially available software (SPSS, Version 19.0; IBM, Armonk, New York) to compare the MR imaging and pathologic data of different grades of glioma from patient group 1. Due to part of the data not being well-represented by a normal distribution, the results have been expressed as mean $\pm \mathrm{SD}$ or median \pm quartile range. The Kruskal-Wallis $\mathrm{H}$ test was used to evaluate differences in the VSI $_{\text {mean }}$ values, microvascular diameter, MVA, and MVD in patients with glioma of different grades, and the Nemenyi method of the multiple comparison was performed. Multiple comparisons between groups were corrected by using the Bonferroni method. The ANOVA and the Least Significant Difference test were used to analyze differences in $\mathrm{VSI}_{\max }$ and $\mathrm{rCBV}_{\max }$ values in patients with glioma of different grades. $P$ values $<.05$ were statistically significant. Pearson correlation analysis was used to evaluate the relationship among VSI values, $\mathrm{rCBV}_{\max }$ values, microvascular diameter, MVA, and MVD. Receiver operating characteristic curves were used to assess the specificity and sensitivity of the $\mathrm{VSI}_{\text {max }}, \mathrm{VSI}_{\text {mean }}$, and $\mathrm{rCBV}_{\text {max }}$ values in distinguishing LGG from HGG. Interrater reliability between the 2 independent VSI observers and the two measurements of interobserver was assessed by using an intraclass correlation coefficient.

\section{RESULTS}

\section{Patient Population}

Overall, 70 of 117 patients participated in our study. Thirty patients (15 women and 15 men; median age, 42 years; age range, $18-79$ years) met the inclusion criteria of group 1, in which there were 9 low-grade ( 3 diffuse astrocytomas, grade II; 5 oligodendrogliomas, grade II; 1 oligoastrocytoma, grade II) and 21 HGGs. Among the high-grade tumors, 8 were diagnosed as grade III (3 anaplastic oligodendrogliomas, 2 anaplastic oligoastrocytomas, 3 anaplastic astrocytomas), and 13 , as grade IV. In addition, we enrolled 20 patients into groups 2 and 3, respectively (On-line Table 1).

AJNR Am J Neuroradiol 37:51-57 Jan 2016 www.ajnr.org 

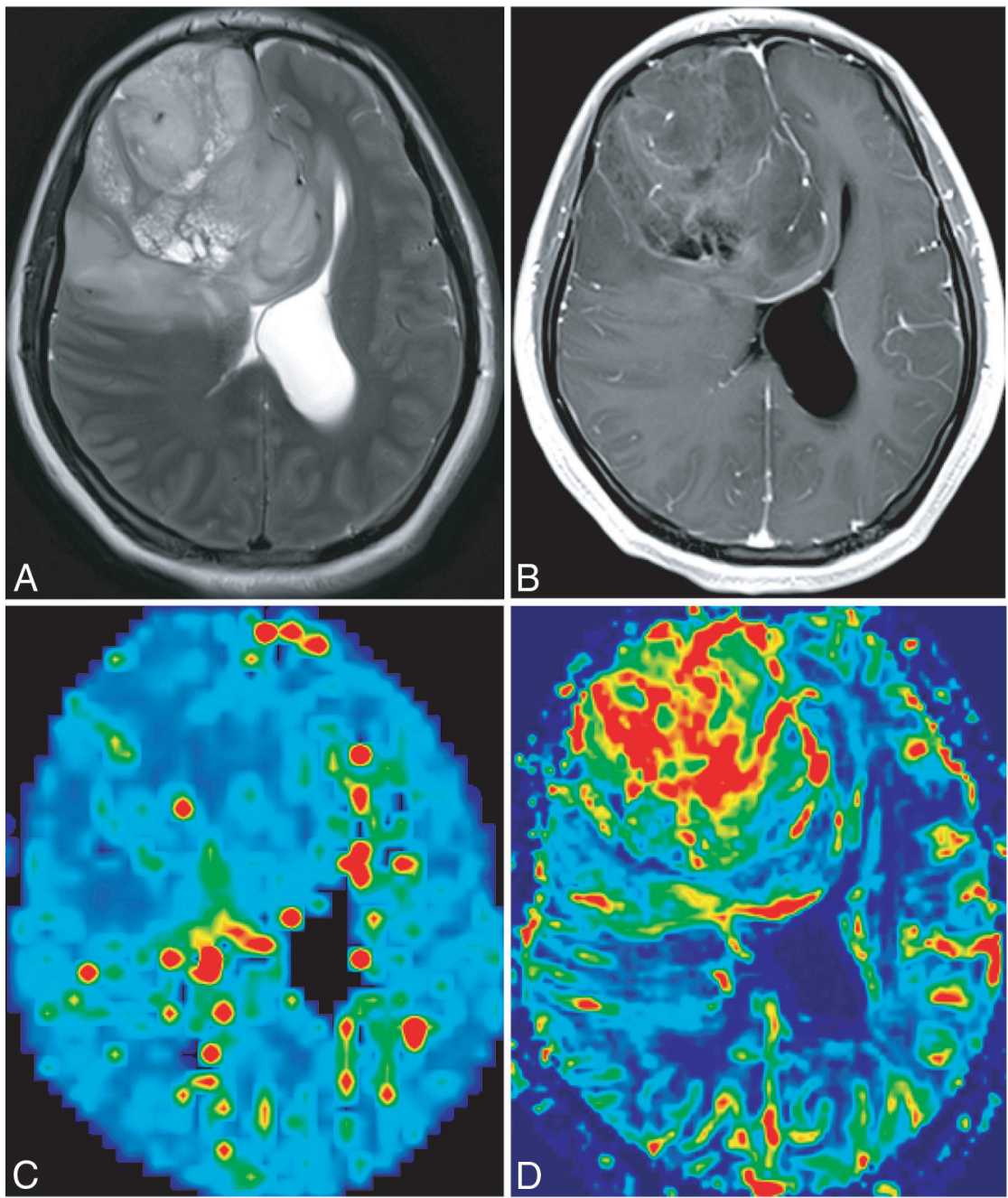

FIG 1. A 25-year-old woman with an oligodendroglioma (World Health Organization grade II). A, Axial T2-weighted MR imaging demonstrates a right frontal and temporal lobe lesion with mass effect, a small amount of edema, and signal-intensity heterogeneity. B, Contrast-enhanced T7-weighted imaging demonstrates mild enhancement. C, The VSI map shows homogeneous low VSI values and is represented in blue-green. $D, C B V$ map demonstrates clearly elevated perfusion in the tumor parenchyma.

\section{Characteristics of VSI and rCBV in Gliomas with Different Grades}

Based on the reference standard, there was excellent intraobserver and interobserver agreement for VSI ${ }_{\text {mean }}$ (intraclass correlation coefficient $>0.879$ ) and VSI ${ }_{\max }$ (intraclass correlation coefficient $>0.809) .{ }^{1}$ The final step was a calculation of the mean values generated from the 4 measurements made by the 2 independent observers. The VSI color map of grade II gliomas is represented in blue-green, denoting low VSI values VSI $_{\text {mean }}$ and VSI ${ }_{\text {max }}$ values, $42.96 \pm 26.43 \mu \mathrm{m}$ and $79.10 \pm 29.17 \mu \mathrm{m}$, respectively); however, the CBV maps of the 2 cases of grade II oligodendrogliomas are represented in red (Fig 1). The VSI and CBV colors maps of grade III and IV gliomas were inhomogeneous and are represented in red, denoting high VSI ${ }_{\text {mean }}$ values (glioma grade III, $130.89 \pm$ $54.74 \mu \mathrm{m}$, and glioblastoma, $154.72 \pm 13.64 \mu \mathrm{m})$, VSI ${ }_{\max }$ values (glioma grade III, $187.44 \pm 43.63 \mu \mathrm{m}$, and glioblastoma, $212.26 \pm 19.69 \mu \mathrm{m}$ ), and rCBV (glioma grade III, $9.15 \pm 5.11$, and glioblastoma, $10.78 \pm 3.66$ ). Although the high perfusion region of CBV partially overlapped that of VSI, the highest perfusion region did not match entirely (Fig 2 ).
Role of VSI and rCBV in Grading Gliomas and Receiver Operating Characteristic Analysis

We found that the VSI ${ }_{\text {mean }}$ and VSI ${ }_{\text {max }}$ values and $\mathrm{rCBV}$ of grade III or IV gliomas were significantly higher than those of grade II gliomas $(P<.01)$, whereas no significant differences were found in $\mathrm{rCBV}$ and the $\mathrm{VSI}_{\text {mean }}$ and VSI $\mathrm{max}_{\text {max }}$ values between gliomas with grades III and IV (Online Fig $1 A-C$ ). To further explore the role of VSI values and $\mathrm{rCBV}$ in distinguishing LGG (grade II) from HGG (grade III and IV), we analyzed the sensitivity and specificity of the VSI mean $_{\text {an }}$ and VSI $I_{\text {max }}$ values and $\mathrm{rCBV}$ by using receiver operating characteristic analysis. At the optimal cutoff VSI ${ }_{\text {mean }}$ values of $99.93 \mu \mathrm{m}$ with the value of sensitivity + specificity- 1 considered maximal, the sensitivity and specificity were both $100 \%$. When the VSI ${ }_{\max }$ values were at the optimal cutoff of $138.3 \mu \mathrm{m}$, the sensitivity and specificity were $95.24 \%$ and $100 \%$, and the AUC value was 0.9947 (On-line Fig $1 D,-E$ ). However, rCBV values at an optimal cutoff of 5.73 , with just $85.71 \%$ sensitivity and $88.89 \%$ specificity, differentiated grade II gliomas from grade III or IV gliomas (On-line Fig 1F).

\section{Histopathologic Characteristics}

From the 30 subjects we enrolled, we collected a total of 75 paraffin blocks, which were used for both histopathologic diagnosis and microvascular quantification. The results revealed that the microvascular diameters and MVA of grade II gliomas were smaller $(9.74 \pm$ $6.17 \mu \mathrm{m}$ and $\left.3365.00 \pm 3287.34 \mu \mathrm{m}^{2}\right)$ than those of grade III gliomas $(23.93 \pm 9.90 \mu \mathrm{m}$ and 18,236.00 \pm $\left.7543.25 \mu \mathrm{m}^{2}\right)$ and grade IV gliomas $(40.48 \pm 18.30 \mu \mathrm{m}$ and $\left.18,196.00 \pm 7534.50 \mu \mathrm{m}^{2}\right)$. The microvessel diameter and MVA of grade III and IV gliomas were significantly higher than those in grade II gliomas $(P<.01)$. The MVD values of grade IV gliomas were significantly higher than those of grade II gliomas $(P<.01)$; however, no significant differences were observed between grade II and III gliomas or grade III and IV gliomas (On-line Fig 2). Furthermore, the microvascular diameters of several grade II oligodendrogliomas were generally smaller than those of diffuse astrocytomas. However, the MVD and MVA of grade II oligodendrogliomas were larger than those of astrocytomas (data not shown).

\section{Correlation between MR Imaging and Microvessel Parameters in Gliomas}

Correlations between MVA and VSI $I_{\text {mean }}$ values, MVA and VSI ${ }_{\text {max }}$ values, MVA and rCBV, microvessel diameter and VSI ${ }_{\text {mean }}$ values, microvessel diameter and VSI $I_{\max }$ values, and microvessel di- 

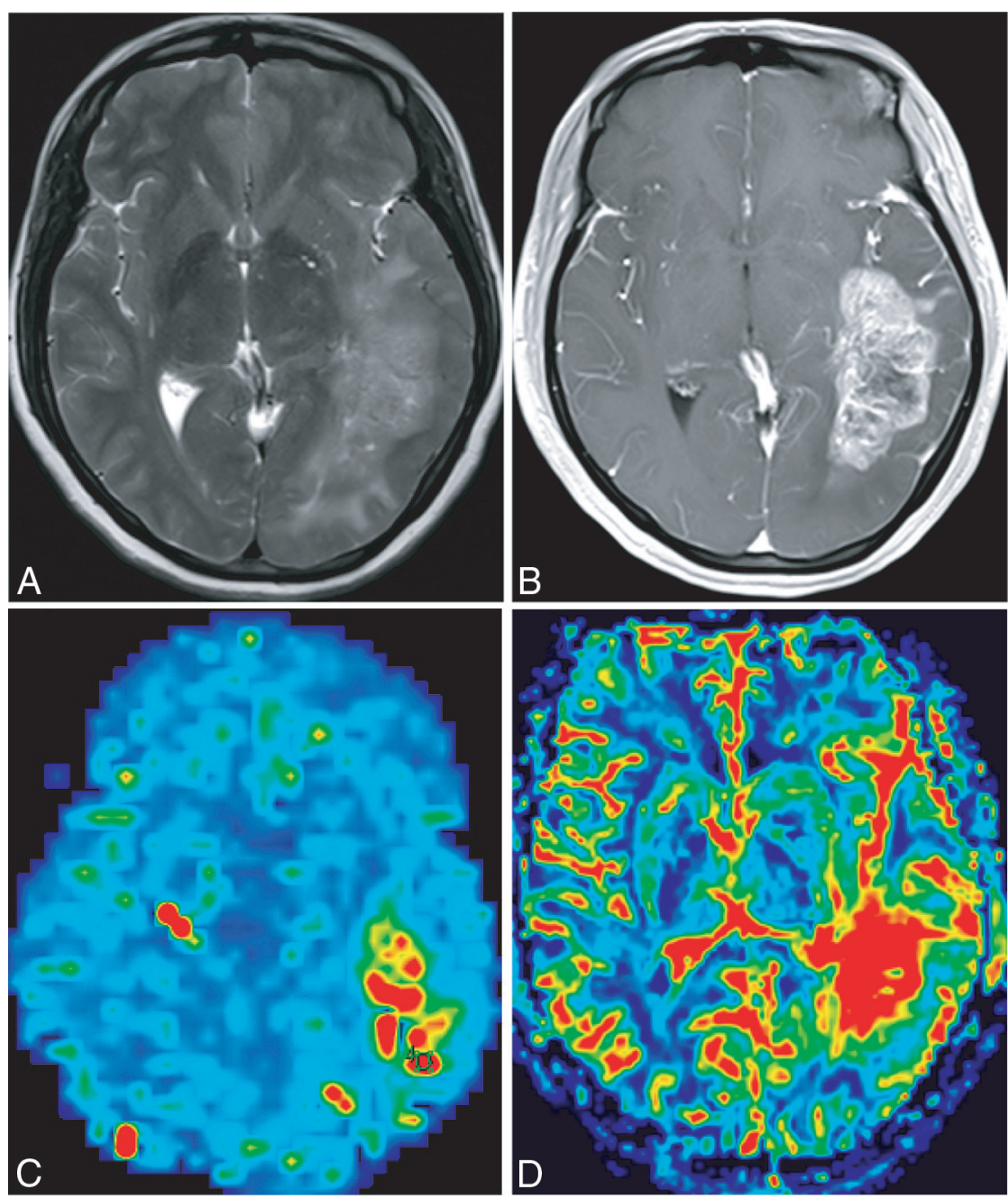

FIG 2. A 42-year-old woman with glioblastoma (World Health Organization grade IV). A, Axial T2-weighted MR imaging reveals a left temporal and occipital lobe lesion with mass effect, a moderate amount of edema, and homogeneous signal intensity. B, Contrast-enhanced T1weighted imaging reveals marked enhancement. C, VSI map shows high VSI values. $D$, CBV map reveals the elevated perfusion more extensively. The highest perfusion region of CBV does not match entirely that of VSI.

ameter and rCBV were all statistically significant $(P<.01)$. Comparing MR imaging and microvessel parameters, we observed the strongest correlation between $\mathrm{VSI}_{\max }$ values and microvascular diameter $(r=0.831)$, followed by VSI ${ }_{\text {mean }}$ values and microvascular diameter $(r=0.824)$. Moreover, no clear correlation could be identified between $\mathrm{CCBV}$ and MVD or between MVD and VSI values (On-line Fig 3).

\section{VSI and rCBV Values as Predictive Factors for Glioma Grading}

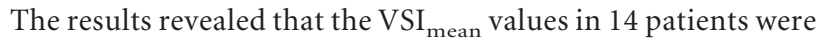
higher than the cutoff value $(99.93 \mu \mathrm{m})$, whereas those in 6 patients were lower. The rCBV values in 13 patients were higher than the cutoff value (5.73), whereas those in 7 patients were lower. Compared with diagnosis by histology (On-line Table 1), as expected, the prediction of VSI for glioma grade had a $100 \%$ accuracy, whereas the accuracy of rCBV was $85 \%$, in which 2 low-grade oligodendrogliomas had $\mathrm{rCBV}$ values higher than the cutoff value and 1 anaplastic astrocytoma was lower than the cutoff value (Fig 3 ).

\section{DISCUSSION}

In this study, we evaluated the sensitivities and specificities of $\mathrm{rCBV}$ and VSI in distinguishing LGGs from HGGs, and we obtained the optimal cutoff values of rCBV and VSI for glioma grading. We found that VSI had a higher accuracy rate in predicting glioma grading compared with rCBV. In addition, we measured microvascular diameter, MVD, and MVA by using CD34 to stain vascular endothelial cells. We found that microvascular diameters and MVA were strongly correlated with the glioma grade compared with MVD.

Histopathologically, endothelial proliferation is an important factor in determining the grades of gliomas. ${ }^{28}$ In recent years, several studies have indicated that tumor grades were strongly correlated with microvessel caliber and MVA, but MVD revealed a poor association with tumor grades. ${ }^{25}$ Consistent with this evidence, our results confirm that microvascular diameters and MVA could differentiate LGGs from HGGs, but MVD only distinguished grade II from grade IV gliomas. These findings suggest that microvessel structure has better efficacy in the grading of gliomas. Furthermore, the comparisons among rCBV, VSI, and histologic results were performed together in the same patients, and the results showed that VSI had better efficacy in reflecting the histologic features of glioma microvessels.

Although an increasing number of articles have shown that rCBV has a high accuracy rate in predicting glioma grades, ${ }^{3,29}$ oligodendrogliomas were shown to have preferentially high $\mathrm{rCBV}$ values regardless of glioma grade. ${ }^{6}$ In our study, we misdiagnosed 2 cases of low-grade oligodendrogliomas by using the rCBV cutoff value, suggesting that $\mathrm{rCBV}$ has limitations in the grading of gliomas. Most interesting, we found that the VSI ${ }_{\text {mean }}$ cutoff value had $100 \%$ accuracy in differentiating LGG and HGG, suggesting that VSI can be used to predict the oligodendroglioma grade. In addition, we found that grade II oligodendrogliomas had shorter microvessel diameters and higher MVD compared with diffuse astrocytomas, possibly resulting in high rCBV arising from oligodendrogliomas.

VSI was considered to noninvasively describe the pathologic changes of vessels in quantitative terms. ${ }^{17}$ In recent years, for VSI investigation, a large number of studies mainly focused on animal models. For example, Troprès et $\mathrm{al}^{17}$ found that VSI positively 
- high grade

口 low grade

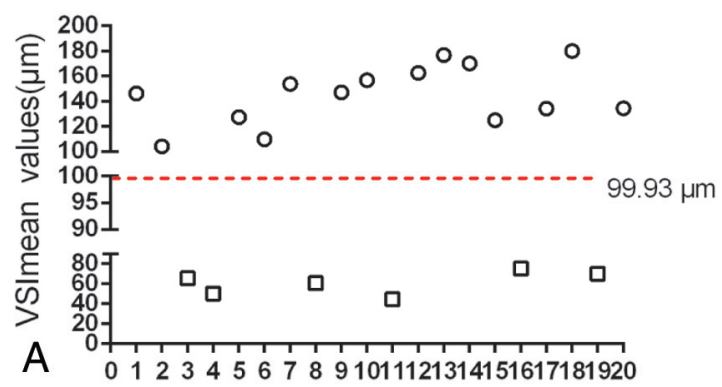

- high grade

口 low grade

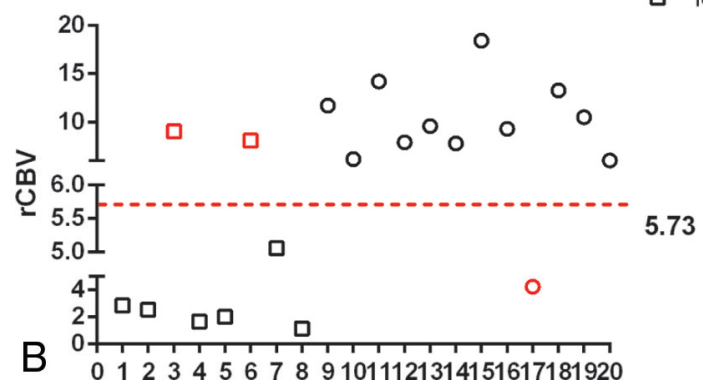

FIG 3. VSI and $r C B V$ values in predicting the grade of gliomas. $A$, The $\mathrm{VSI}_{\text {mean }}$ values show no overlap between HGG and LGG and provide $100 \%$ accuracy in predicting the glioma grade. $B$, The glioma grade could not be exactly identified on the basis of the cutoff values of rCBV. Black indicates the cases correctly identified, and red denotes the cases incorrectly identified.

correlated with the pathologic diagnosis, a Spearman coefficient of 0.74 . Lemasson et al reported that MR imaging and histologic estimates of VSI correlated well and may be used to characterize angiogenesis in vivo beyond CBV, as determined in 27 rats with intracranial gliomas. ${ }^{4}$ Recently, Emblem et al ${ }^{16}$ indicated that vessel architectural imaging had the potential to identify patients who might benefit from certain therapies. However, the application of VSI in human glioma grading remains unclear. In our study, we showed that the VSI values were positively correlated with the histologic results, with a correlation coefficient of $>0.80$, and VSI had a high accuracy rate in predicting glioma grades. In addition, we found that VSI mean values had higher AUC, sensitivity, and specificity for glioma diagnosis than VSI ${ }_{\max }$ values; this outcome may have been the result of the following 2 points: 1 ) $\mathrm{VSI}_{\text {mean }}$ values better reflect the status of whole tumors; and 2) the signal-to-noise ratio of VSI is poor, resulting in a higher rate of errors in the measurement of VSI $\max _{\max }$ values.

Our study had limitations. First, the number of the glioma samples, especially grade II and III gliomas, was modest. Second, robust VSI estimates should be obtained from patients at $3 \mathrm{~T}$ or higher magnetic fields by using the DSC approach. ${ }^{30}$ Third, we used the "hot-spot" method, which cannot ensure the consistency between MR imaging regions and pathology measurements. Fourth, the time and space resolution of VSI was not as good as that of CBV; the signal-to-noise ratio of VSI images required enhancing to demonstrate regions of scattered perfusion in the cerebral tissue or lateral cerebral ventricles. Fifth, the lack of a unified approach to performing VSI measurement may result in unstable VSI cutoff values. In future studies, we will search for more objective and effective methods for VSI measurement.

\section{CONCLUSIONS}

In this study, we compared VSI, rCBV, and histologic results in grading gliomas and showed that VSI has greater accuracy at predicting glioma grading than $\mathrm{rCBV}$, suggesting that VSI may serve as an effective tool for the diagnosis of patients with gliomas and for guiding the clinical therapeutic strategy.

\section{ACKNOWLEDGMENTS}

We thank He Wang, Jing Ning, and Hui Lin for their technical assistance, and San-Gao Fang for the contribution to pathology measurement in this study.

Disclosures: Hou-Yi Kang, Jin-Hua Chen, Yong Tan, Tian Xie, Wei-Guo Zhang-RELATED: Grant: Natural Science Foundation of China (No. 81271626) and Chongqing (No. cstc2012jjB10028), and the Scientific Foundation of Institute of Surgery Research, Daping Hospital, Third Military Medical University (NO. 2014YLC03).* Xiao ChenRELATED: Grant: National Natural Science Foundation of China (grant No. 81271626).* *Money paid to the institution.

\section{REFERENCES}

1. Caulo M, Panara V, Tortora D, et al. Data-driven grading of brain gliomas: a multiparametric MR imaging study. Radiology 2014;272: 494-503 CrossRef Medline

2. Haegler K, Wiesmann M, Böhm C, et al. New similarity search based glioma grading. Neuroradiology 2012;54:829-37 CrossRef Medline

3. Hu LS, Eschbacher JM, Dueck AC, et al. Correlations between perfusion MR imaging cerebral blood volume, microvessel quantification, and clinical outcome using stereotactic analysis in recurrent high-grade glioma. AJNR Am J Neuroradiol 2012;33:69-76 CrossRef Medline

4. Lemasson $B$, Valable $S$, Farion $R$, et al. In vivo imaging of vessel diameter, size, and density: a comparative study between MRI and histology. Magn Reson Med 2013;69:18-26 CrossRef Medline

5. Knopp EA, Cha S, Johnson G, et al. Glial neoplasms: dynamic contrast-enhanced T2*-weighted MR imaging. Radiology 1999;211: 791-98 CrossRef Medline

6. Spampinato MV, Smith JK, Kwock L, et al. Cerebral blood volume measurements and proton MR spectroscopy in grading of oligodendroglial tumors. AJR Am J Roentgenol 2007;188:204-12 CrossRef Medline

7. Law M, Yang S, Babb JS, et al. Comparison of cerebral blood volume and vascular permeability from dynamic susceptibility contrastenhanced perfusion MR imaging with glioma grade. AJNR Am J Neuroradiol 2004;25:746-55 Medline

8. Hilario A, Ramos A, Perez-Nuñez A, et al. The added value of apparent diffusion coefficient to cerebral blood volume in the preoperative grading of diffuse gliomas. AJNR Am J Neuroradiol 2012;33: 701-07 CrossRef Medline

9. Hakyemez B, Erdogan C, Ercan I, et al. High-grade and low-grade gliomas: differentiation by using perfusion MR imaging. Clin $\mathrm{Ra}$ diol 2005;60:493-502 CrossRef Medline

10. Khalid L, Carone M, Dumrongpisutikul N, et al. Imaging characteristics of oligodendrogliomas that predict grade. AJNR Am J Neuroradiol 2012;33:852-57 CrossRef Medline

11. Bian W, Khayal IS, Lupo JM, et al. Multiparametric characterization of grade 2 glioma subtypes using magnetic resonance spectroscopic, perfusion, and diffusion imaging. Transl Oncol 2009;2: 271-80 CrossRef Medline

12. Guillevin R, Menuel C, Abud L, et al. Proton MR spectroscopy in predicting the increase of perfusion MR imaging for WHO grade II gliomas. J Magn Reson Imaging 2012;35:543-50 CrossRef Medline

13. Lin $\mathrm{Y}$, Dawant BM. Automatic detection of the anterior and poste- 
rior commissures on MRI scans using regression forests. Conf Proc IEEE Eng Med Biol Soc 2014;2014:1505-08 CrossRef Medline

14. Roy B, Gupta RK, Maudsley AA, et al. Utility of multiparametric 3-T MRI for glioma characterization. Neuroradiology 2013;55:603-13 CrossRef Medline

15. Boxerman JL, Hamberg LM, Rosen BR, et al. MR contrast due to intravascular magnetic susceptibility perturbations. Magn Reson Med 1995;34:555-66 CrossRef Medline

16. Emblem KE, Mouridsen K, Bjornerud A, et al. Vessel architectural imaging identifies cancer patient responders to anti-angiogenic therapy. Nat Med 2013;19:1178-83 CrossRef Medline

17. Troprès I, Lamalle $\mathrm{L}$, Péoc'h $\mathrm{M}$, et al. In vivo assessment of tumoral angiogenesis. Magn Reson Med 2004;51:533-41 CrossRef Medline

18. Kiselev VG, Strecker R, Ziyeh S, et al. Vessel size imaging in humans. Magn Reson Med 2005;53:553-63 CrossRef Medline

19. Hsu YY, Yang WS, Lim KE, et al. Vessel size imaging using dual contrast agent injections. J Magn Reson Imaging 2009;30:1078-84 CrossRef Medline

20. Choi SH, Chi JG, Kim YB, et al. Anterior commissure-posterior commissure revisited. Korean J Radiol 2013;14:653-61 CrossRef Medline

21. Jerome NP, Hekmatyar SK, Kauppinen RA. Blood oxygenation level dependent, blood volume, and blood flow responses to carbogen and hypoxic hypoxia in 9L rat gliomas as measured by MRI. J Magn Reson Imaging 2014;39:110-19 CrossRef Medline

22. Hu LS, Baxter LC, Pinnaduwage DS, et al. Optimized preload leakage-correction methods to improve the diagnostic accuracy of dynamic susceptibility-weighted contrast-enhanced perfusion MR imaging in posttreatment gliomas. AJNR Am J Neuroradiol 2010;31: 40-48 CrossRef Medline
23. Paulson ES, Schmainda KM. Comparison of dynamic susceptibilityweighted contrast-enhanced MR methods: recommendations for measuring relative cerebral blood volume in brain tumors. Radiology 2008;249:601-13 CrossRef Medline

24. Emblem KE, Nedregaard B, Nome T, et al. Glioma grading by using histogram analysis of blood volume heterogeneity from MR-derived cerebral blood volume maps. Radiology 2008;247:808-17 CrossRef Medline

25. Deb P, Boruah D, Dutta V. Morphometric study of microvessels in primary CNS tumors and its correlation with tumor types and grade. Microvasc Res 2012;84:34-43 CrossRef Medline

26. Korkolopoulou P, Patsouris E, Konstantinidou AE, et al. Hypoxiainducible factor 1alpha/vascular endothelial growth factor axis in astrocytomas: associations with microvessel morphometry, proliferation and prognosis. Neuropathol Appl Neurobiol 2004;30:267-78 CrossRef Medline

27. Sharma S, Sharma MC, Sarkar C. Morphology of angiogenesis in human cancer: a conceptual overview, histoprognostic perspective and significance of neoangiogenesis. Histopathology 2005;46: 481-89 CrossRef Medline

28. Daumas-Duport C, Scheithauer B, O'Fallon J, et al. Grading of astrocytomas: a simple and reproducible method. Cancer 1988;62: 2152-65 Medline

29. Lemasson B, Chenevert TL, Lawrence TS, et al. Impact of perfusion map analysis on early survival prediction accuracy in glioma patients. Transl Oncol 2013;6:766-74 CrossRef Medline

30. Pannetier N, Lemasson B, Christen $\mathrm{T}$, et al. Vessel size index measurements in a rat model of glioma: comparison of the dynamic (Gd) and steady-state (iron-oxide) susceptibility contrast MRI approaches. NMR Biomed 2012;25:218-26 CrossRef Medline 